\title{
TEXTTB: A PARALLEL DESIGN RANDOMIZED CONTROL PILOT STUDY TO EVALUATE ACCEPTANCE AND FEASIBILITY OF A PATIENT-DRIVEN MOBILE PHONE BASED INTERVENTION TO SUPPORT ADHERENCE TO TB TREATMENT
}

\begin{tabular}{|c|c|c|}
\hline \multicolumn{3}{|c|}{$\begin{array}{c}\text { Sarah Iribarren, }{ }^{\mathbf{1}} \text { Christina Chirico, }^{\mathbf{2}} \text { Mirta Echevarrria, }{ }^{\mathbf{2}} \text { Daniel Cardinali }{ }^{\mathbf{2}} \\
{ }^{1} \text { University of Utah, College of Nursing, USA, }{ }^{2} \text { Health Region V TB Program, Hospital Dr A Cetrangolo, Argentina }\end{array}$} \\
\hline Journal MTM 1:4S:23-24 & DOI: 10.7309/jmtm.46 & www.journalmtm.com \\
\hline
\end{tabular}

\section{ABSTRACT}

BACKGROUND Argentina's treatment success rate remains well below the World Health Organization (WHO) TB key target of $85 \%$, averaging 46\% from 2008-2010. Few studies have applied mobile phone-based interventions for TB management. This study assessed feasibility, acceptance and explored the initial efficacy of a short message service (SMS)-based intervention to improve patient adherence and support TB patients in a setting where self-administration is the standard care.

METHODS Newly diagnosed TB patients were screened from November 2011 - September 2012 from a hospital-based outpatient clinic within a pulmonary specialized reference hospital in Argentina. Patients were randomized into control and intervention in a 1:1 allocation ratio in block randomization of 10 . The intervention arm received standard of care plus a SMS-based intervention which included instructing patients to 'text-in' after self-administration of medication; reminders/check-in when patient did not 'text in'; receipt of bi-weekly SMS education messages; and the option to consult during the first two month intensive treatment phase. Educational messages were selected based on the Informational-Motivational-Behavioral Skills Model. FrontlineSMS opensource software was used as the SMS platform. Semi-structured, in depth, individual interviews were conducted with 9 patients and 3 others responded to structured questions via text-messages. Outcomes of feasibility (e.g. number of potential participants with mobile phones), acceptability (e.g. participant and staff perceptions, type of messages) and initial efficacy (e.g. notification rates, sputum conversion) were assessed.

RESULTS Thirty-seven participants were enrolled from 122 screened, of whom 3 potential participants did not have access to a phone and 3 did not know how to send text message. Other feasibility issues included: varying mobile phone coverage (58\% interviewed notified having some technical difficulties, e.g. missed sent/received messages), modem partially capturing data, feature limitations of FrontlineSMS (e.g. manual individual entry of each educational message, time stamp 2 hours early), computer security and challenges with infrastructure/staffing to track and bring patients who did not notify back to treatment. Most participants found intervention acceptable ( $82 \%$ would highly recommend to others), reported being "cared for" and "having a friend when all others wanted nothing to do with them" and that notifying made them feel that they were "responsible for their treatment". Four participants were not included in final analysis (1 transferred care, 1 withdrew, 2 due to modem technical problems). Average notification rate $(\mathrm{n}=14)$ over 60 -day period was $77 \%(22-100 \%)$, of those $83 \%(53-100 \%)$ notified without reminder. Running out of credit and technical difficulties were reported as reasons for missing notification. On average, participants texted in 2 questions (2-6) and 1.5 messages that reported side-effects (0-8). Only $15(45 \%)$ had follow-up sputum smear or culture tests, the difference between groups was non-significant.

CONCLUSIONS Mobile phones and text messaging was prevalent among potential participants making intervention contextually appropriate in this setting. Intervention was largely accepted. Feasibility issues may be 
יJMTM

improved with adjunct programs to manage educational texts, free-to-text in number and strengthening infrastructure to trace and bring patients back to treatment. Treatment outcomes, such as sputum-smear or culture, testing is low. Further research is needed to evaluate this interactive intervention's potential. 